\title{
Christian K. Schneider
}

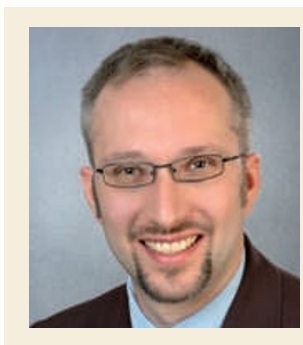

Chair, Committee for Advanced Therapies (CAT), European Medicines Agency (EMEA), London, UK. Christian K. Schneider, M.D., is currently Head of Division of EU Co-operation/Microbiology at the Paul Ehrlich Institute, the German Federal Agency for Sera and Vaccines. He is a co-opted member of the Committee for Medicinal Products for Human Use (CHMP) at the EMEA, and the chairman of the CHMP Working Party on Similar Biological Medicinal Products. In February, he was elected Chair of the EMEA's CAT, which was created when the new European Union legislation on advanced therapies came into force in December 2008.

Please could you define advanced therapies and explain why CAT has been created? Advanced therapies are divided into three categories of products: gene therapy medicinal products, somatic cell therapies and tissue engineered products. Such treatments are very promising, but to make them available to patients safely - and ensure they are effective - requires specific expertise. It was found that the current committees mainly have broad expertise in classical biological and chemical drugs, and so the European Commission (EC) decided to create CAT to provide guidance for product development and to assess marketing authorization applications (MAAs) for advanced therapies.

In addition, the EC wanted Europe to be a more fruitful environment for developers of these products - many of which are academic researchers or start-up companies. To help such developers, a new procedure has been invented called the certification procedure. Companies can now ask for a certificate for their quality development, which includes their chemistry, manufacturing and controls, and their non-clinical development. Although the conduct of the procedure is still under discussion, this certificate might be an incentive for a bigger company to buy the patent or invest in the development of an advanced therapy medicinal product created by academic researchers or smallor medium-sized enterprises.

\section{What are the main objectives of CAT?}

We aim to ensure that EC-approved products are high quality, safe and efficacious. This seems like a standard answer, but many of the companies creating advanced therapies are new and lack knowledge of the regulatory requirements. One of the main objectives is therefore to provide expert advice for the development and licensing of advanced therapies in Europe. There is a principle in EU law that says that the EMEA and its committees shall make the best use of available expertise in Europe. CAT will therefore take part in the scientific advice procedures, to give specific input to the experts in the EMEA Scientific Advice Working Party who have experience in the more classical fields of pharmacy and product development. CAT will also lead and perform the assessment during the MAA procedure and prepare the draft opinion for the CHMP — which is responsible by law for giving the scientific opinion to the EC on the recommendation or refusal of an MAA for any product for human medicinal use, which includes advanced therapies.

How might companies developing advanced therapies in Europe benefit from the existence of such a committee?

A big benefit is that CAT provides a regulatory partner. Of course, to do this the new committee needs to establish the possibilities for interaction. Currently, this includes the formal certification and scientific advice procedures. However, there is also an informal exchange by so-called briefing meetings, in which CAT will participate. These were created years ago by the EMEA's Innovation Task Force so that companies can come to the agency for regulatory discussions and scientific exchange at an early stage of their product development (it is not official scientific advice). One of my personal aims is to avoid being regulators in an ivory tower, so that CAT can be approached to find solutions to problems that are inherent to these products.

\section{Could you outline the main challenges you foresee with advanced therapies?}

The main regulatory challenge is the need to demonstrate that an advanced therapy is high quality, safe and effective, using methods that are applicable for each specific product. For example, with a cell-based therapy it is not always possible to define a product in the same way that a drug would be characterized, such as determining all aspects of the mechanism of action or the pharmacokinetics. However, it is important that different regulatory standards are not set. To avoid this, it will be important for other committees, who have considerable expertise in their fields, to provide their input.

Moreover, pre-clinical development of biotechnology products requires a relevant animal model for toxicity testing. But for advanced therapies, the products are sometimes so human specific that not even higher monkeys are relevant for testing. To address this, developers create a surrogate product to test in animals - this is not the product that will be tested in humans. This is one of the biggest challenges for these products.

The other scientific challenge is clinical-trial design. As many advanced therapies aim to address unmet medical needs in indications that currently have no treatment options, there may be ethical challenges for designing that trial. For example, CAT and the developers will have to find new ways of designing trials - to find a comparator or an end point for a disease with no currently available treatment.

\section{How can regulators, academic researchers} and companies address these challenges? First, the companies and academic researchers need to be aware that they can interact with us. For example, we will hold a stakeholder workshop in April to begin the dialogue.

Second, CAT will consider publishing regulatory and scientific considerations in scientific journals. This is partly because academics and small companies often produce advanced therapies, and the normal way of communicating regulatory issues, such as through the EMEA website, might not be known to them. It is hoped that scientific publications would give the committee a stronger role in the community and ultimately encourage industry and academic groups to approach CAT more readily.

Interview by Bethan Hughes 